\title{
Pulmonary vein thrombosis extending to left atrium after non-small cell lung cancer
}

\author{
Fatih Sivri *, Hasan Güngör, Çağdaş Akgüllü \\ Adnan Menderes University, Department of Cardiology, Aydin, Turkey
}

\section{Introduction}

Pulmonary vein thrombosis (PVT) is a rare disease and it has been described following lobectomy, and lung transplantation. Some cases have been described as idiopathic and most commonly associated with metastatic carcinoma ${ }^{1,2}$. An atypically large thrombus extending from primary lung cancer into the left atrium (LA) is very rare. We report a 63-year-old man with non-small cell cancer presented with a large PVT extending to the LA through the left inferior pulmonary vein.

\section{Case}

A 63-year-old man with non-small cell lung cancer admitted to our emergency department with haemoptysis and dyspnea. On examination, his vital signs were stable and electrocardiography indicated sinus rhythm. Heart sounds were distinct and no murmur was heard. Chest radiograph showed round shaped mass with well-defined margins measuring $6 \mathrm{~cm}$. A computed tomography (CT) scan of chest revealed a mass measuring $4.5 \times 4.0 \mathrm{~cm}$ in lower lob of the left lung and hypodense appearance consistent with thrombus extending from the lung to the left atrium (LA) via a pulmonary vein (Fig. 1). Transthoracic echocardiography (TTE) showed a large round shaped mobile hypoechoic mass in the LA and transesophageal echocardiography (TEE) confirmed the diagnosis. Cardiac magnetic resonance showed a hypodense mass consistent with thrombus extending from the lung to the left atrium via a pulmonary vein (Fig. 2). PET scan showed any cardiac metastasis.

Our patient was consulted with pulmonary department due to the haemoptysis and they recommended tranexamic acid treatment. Surgery was not considered due to life-threatening complications including systemic embolism and sudden death. Patient was followed for a week any anticoagulant treatment cannot be started because of the haemoptysis. One week later we could start low-molecular-weight heparin $(\mathrm{LMWH})$, enoxaparin and patient was followed optimal dose LMWH for four months. Any embolic events were seen during our treatment. After four months CT scan showed that thrombus size was smaller (Fig. 3).

\section{Discussion}

The pathogenesis of PVT from a tumor is unknown and it could result from epithelial damage as a result of tumor invasion, from direct extension of the tumor into the vein and from compression of the vein by the tumor $^{3}$. The symptoms of PVT are usually nonspecific, including dyspnea, cough, pleuritic chest pain, and haemoptysis. It has been reported as causing massive haemoptysis due to acute pulmonary infarction ${ }^{4}$. A free-floating PVT extending to the LA is an unusual metastasis of nonsmall cell lung cancer. CT scan imaging and TTE are more accurate in the diagnostic process of these patients ${ }^{5}$. There are no standard treatments for this particular patient, surgery is preferred especially in large embolus formation. Long-term anticoagulation can be chosen as an alternative treatment in patients with high-risk.

\section{Conflict of interest}

None.

\section{References}

1. Tamizifar B, Zadeh MR, Foroghi E. Pulmonary vein thrombosis after metastatic liposarcoma. Med Arh 2012;66:68-69.

2. Akiode O, Pragash G. Pulmonary vein thrombosis associated with metastatic carcinoma. Fed Pract 2014;31:26-28.

3. Wu JP, Wu Q Yang Y, DU ZZ, Sun HF. Idiopathic pulmonary vein thrombosis extending to left atrium: a case report with a literature review. Chin Med J 2012;125:1197-1200.

4. Alexander GR, Reddi A, Reddy D. Idiopathic pulmonary vein thrombosis: a rare cause of massive hemoptysis. Ann Thorac Surg 2009;88:281-283.

5. Lin MT, Ku SC, Wu MZ. Intracardiac extension of lung cancer via the pulmonary vein. Thorax 2008;63:1122.

\footnotetext{
* Corresponding author at: Adnan Menderes University, Faculty of Medicine Department of Cardiology, 09100 Aydin, Turkey.

E-mail address: fatih1991sivri@gmail.com (F. Sivri)

Peer review under responsibility of The Society of Cardiovascular Academy.
} 


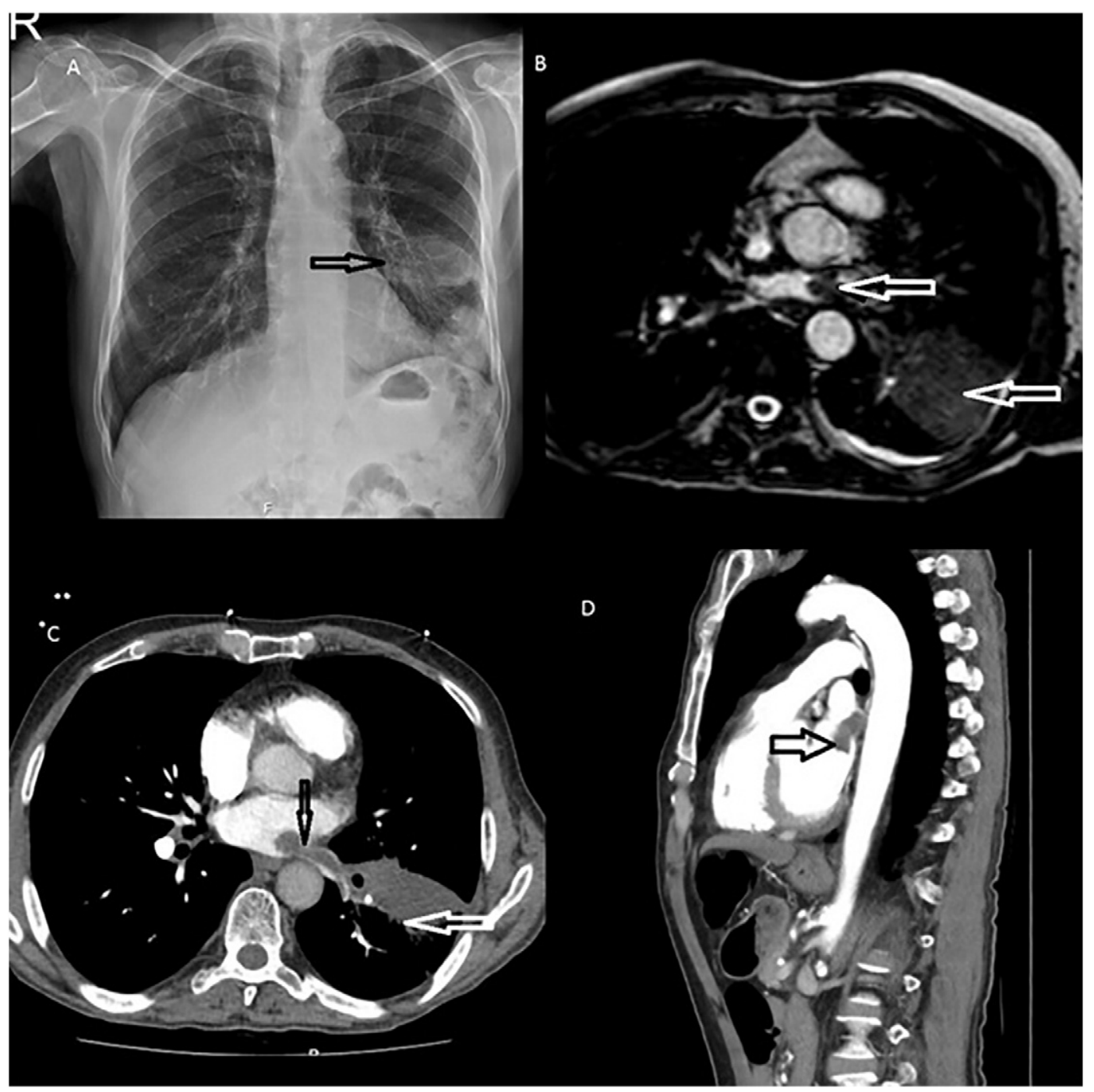

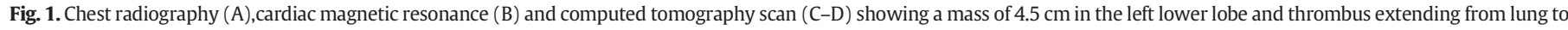
the left atrium via pulmonary vein. 


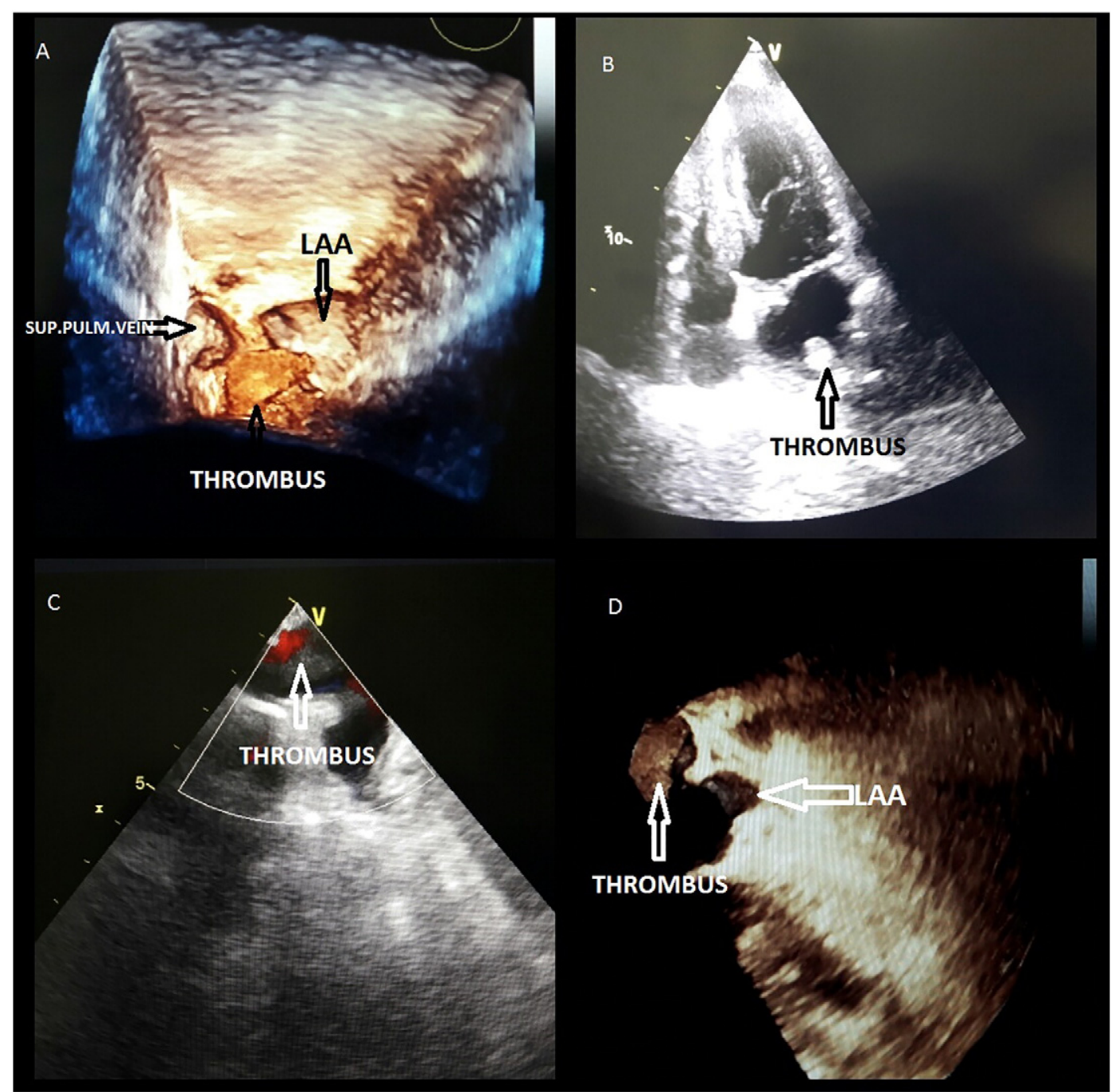

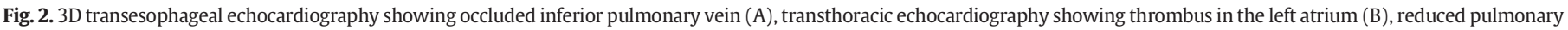
vein flow $(C)$ and left atrial appendage without thrombus (D). 

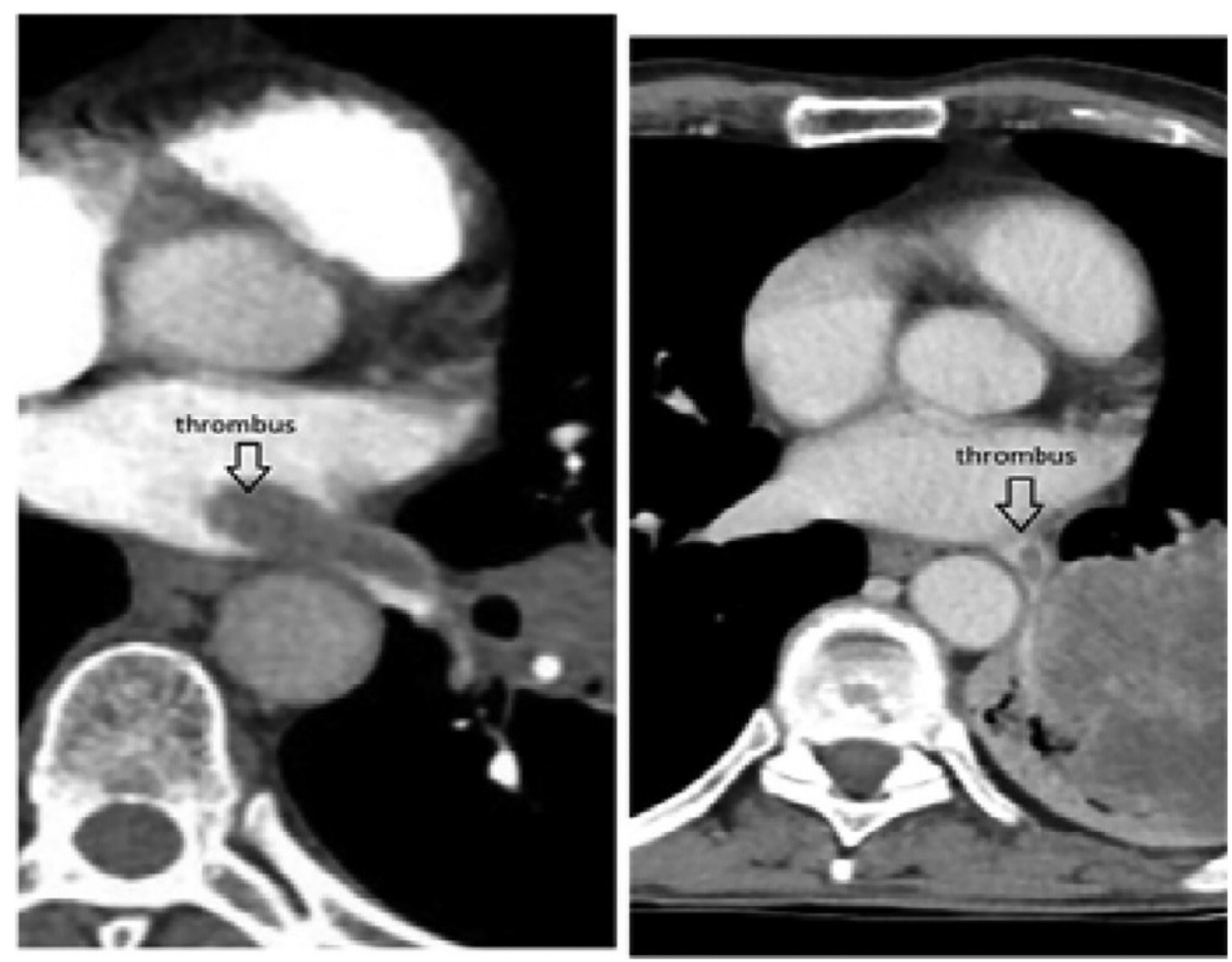

Fig. 3. Regression of the thrombus after treatment. 